\title{
Health behaviors and metabolic risk factors are associated with dyslipidemia in ethnic Miao Chinese adults: the China multi-ethnic cohort study
}

Fang Nie ${ }^{1,2}$, Ziyun Wang ${ }^{1}$, Qibing Zeng ${ }^{1}$, Han Guan ${ }^{1}$, Jingyuan Yang ${ }^{1}$, Peng Luo ${ }^{1}$, Lunwei Du ${ }^{1,2}$, Junhua Wang ${ }^{1 *}$ and Feng Hong ${ }^{1 *}$

\begin{abstract}
Background: Cardiovascular risk factors in Chinese ethnic minority groups are rarely reported.

Objective: To quantify the cardiovascular risk factors in Miao Chinese adults and to examine the association of health behaviors and metabolic risk factors with dyslipidemia.
\end{abstract}

Methods: A cross-sectional analysis was conducted using baseline data from the China Multi-Ethnic Cohort (CMEC) study. A representative sample of 5559 Miao participants aged 30 to 79 years were surveyed and given physical and laboratory exams. The proportion of behavioral and metabolic risk factors were described in ethnic Miao adults. Logistic regression was utilized to evaluate the odds ratio (OR) and 95\% confidence interval (Cl) of the association between health behaviors and metabolic risk factors with dyslipidemia.

Results: In Miao Chinese adults, the prevalence of dyslipidemia was 32.8\%. After multivariate adjustment, subjects with poor waist-to-hip ratio (WHR), body mass index (BMI), fasting blood glucose (FBG) and blood pressure (BP) were more likely to have higher risk of triglycerides (TG) abnormality, regardless of gender and age. Furthermore, the strongly association was detected between poor WHR and low density lipoprotein cholesterol (LDL-C) abnormality (adjusted $\mathrm{OR}=5.24,95 \% \mathrm{Cl}: 2.42-11.34$ ) in the older subgroup ( $\geq 60$ years). Males who current smoking were an independent risk factor only for high density lipoprotein cholesterol (HDL-C) abnormality (adjusted OR= 1.44, 95\%Cl: 1.05-1.99). However, in the subgroup age, current smoker were at greater risk of high TG and low HDLC. Males with regular drinking were less likely to be high $L D L-C$ (adjusted $O R=0.51,95 \% C l: 0.32-0.81$ ).

Conclusions: The present findings indicated that Miao adults with metabolic risk factors were at greater risk of dyslipidemia.

Keywords: Dyslipidemia, Cardiovascular health, Health behaviors, Metabolic risk factors, Minorities

\footnotetext{
* Correspondence: 740064265@qq.com; 519490967@qq.com

'School of Public Health, The Key Laboratory Of Environmental Pollution Monitoring and Disease Control, Ministry of Education, Guizhou Medical University, Guiyang 550025, China

Full list of author information is available at the end of the article
}

C C The Author(s). 2021 Open Access This article is licensed under a Creative Commons Attribution 4.0 International License, which permits use, sharing, adaptation, distribution and reproduction in any medium or format, as long as you give appropriate credit to the original author(s) and the source, provide a link to the Creative Commons licence, and indicate if changes were made. The images or other third party material in this article are included in the article's Creative Commons licence, unless indicated otherwise in a credit line to the material. If material is not included in the article's Creative Commons licence and your intended use is not permitted by statutory regulation or exceeds the permitted use, you will need to obtain permission directly from the copyright holder. To view a copy of this licence, visit http://creativecommons.org/licenses/by/4.0/ The Creative Commons Public Domain Dedication waiver (http://creativecommons.org/publicdomain/zero/1.0/) applies to the data made available in this article, unless otherwise stated in a credit line to the data. 


\section{Background}

Cardiovascular disease (CVD) is a public health problem with high morbidity and mortality and accounts for over $40 \%$ of deaths from all causes [1]. Dyslipidemia is a wellestablished risk factor for CVD [2]. Elevated total cholesterol (TC) and low density lipoprotein cholesterol (LDLC) abnormality are generally considered to be independent risk factors for atherosclerosis [3]. Studies showed that for each $1 \mathrm{mmol} / \mathrm{L}$ decrease in LDL-C, this risk of cardiovascular event is reduced by $21 \%$ [4]. In clinical practice, these metabolic signs are the most commonly used indicators of CVD risk [5, 6].

Epidemiological data show that $64.4 \%$ of the Chinese population has at least one type of dyslipidemia [7]. The high prevalence of dyslipidemia is related to a dramatic shift to an unhealthy lifestyle [8]. Prior study has reported that the lipid metabolism is closely related to lifestyle factors $[9,10]$. Multiple healthy lifestyle may be as effective as drug therapy at preventing dyslipidemia, and these lifestyle factors are better managed and moderated [11]. Therefore, the increased prevalence of dyslipidemia is likely attributable to lifestyle risk factors.

In Guizhou province, the Miao ethnic group accounts for $42.1 \%$ of all Miao people according to the sixth census [12]. The Miao people have deep historic roots and still maintain old customs and a unique lifestyle that are distinct from the majority of Han Chinese [13]. Although dyslipidemia is well documented by studies in the Han people, the evidence poorly understood in ethnic Chinese minority groups and the practice of dyslipidemia management is still limited. Consequently, to better understand the association between health behaviors and metabolic risk factors with dyslipidemia, we conducted an analysis using baseline data from the China Multi-Ethnic Cohort (CMEC) study.

\section{Materials and methods Study participants}

The CMEC study is a large-scale prospective cohort study based on community population in five provinces of southwest China, Guizhou, Yunnan, Sichuan, Chongqing and Tibet. From July 2018 to April 2019, 99,556 members aged 30 to 79 years old were recruited. Further details are available elsewhere [14].

Multi-stage stratified random sampling was used to select the study participants. The Miao and Dong autonomous prefecture of Qiandongnan, and the Bouyei and Miao autonomous prefecture of Qiannan were selected based on the characteristics of ethnic minority groups in Guizhou. Next, from these prefecture, Kaili City, Liping County, and Libo County were selected as secondary sampling units.

\section{Questionnaire and physical examinations}

More detailed information on questionnaire and medical examinations are published elsewhere [14]. In brief, information on health behaviors was collected by questionnaire in face-to-face interviews with trained medical student. Behavioral data included: smoking status, alcohol consumption status, average sleep duration, and physical activity (PA). PA was calculated as the total of the metabolic equivalent of each task in hours per day.

Blood sample was collected by professional nurses from participants after a mandatory 8-h fast. Samples were sent to the JinYu Medical Laboratory Center of Guizhou Province for analysis of TC, LDL-C, high density lipoprotein cholesterol (HDL-C), triglycerides (TG), and fasting blood glucose (FBG).

$\mathrm{BP}$ was recorded as the average of three measurements taken at 5-min intervals using an electronic sphygmomanometer. WHR was calculated from the ratio of waist circumference to hip circumference. Waist and hip circumference were measured using soft tape. BMI was defined as weight in kilograms divided by the square of the height in meters $\left(\mathrm{kg} / \mathrm{m}^{2}\right)$.

Participant responses were categorized as Ideal, Intermediate, or Poor based on definitions listed in Table 1. The categories were defined by guidelines form the American Heart Association combined with characteristics specific to Chinese people $[15,16]$.

\section{Dyslipidemia outcomes}

According to published guidelines for Chinese adults [17], dyslipidemia was defined the presence of any of these four indicators: $\mathrm{TC} \geq 6.22 \mathrm{mmol} / \mathrm{L}, \mathrm{TG} \geq 2.26 \mathrm{mmol} / \mathrm{L}$, LDL$\mathrm{C} \geq 4.14 \mathrm{mmol} / \mathrm{L}$, or HDL-C $<1.04 \mathrm{mmol} / \mathrm{L}$.

\section{Statistical analysis}

Categorical variables were expressed as $\mathrm{n}$ (\%). The demographic characteristics between different groups were compared using $x^{2}$ test for categorical variables. The distribution of health behaviors and metabolic risk factors were summarized by gender, age and place of residence. The prevalence of dyslipidemia was stratified by gender. We also described the prevalence by each component of lipid. The age-standardized prevalence of dyslipidemia was calculated corresponding to the sixth census of Guizhou in 2010.

Univariate and multivariate logistic regression was used to evaluate the association between health behaviors and metabolic risk factors with dyslipidemia components, stratified by gender. High TC, high TG, high LDL-C and low HDL-C were used as the dependent variables. The regression model was adjusted for age, area of residence, education, occupation, marital status and family income as defined in Table 2 . The analysis was also stratified to subjects $<60$ and $\geq 60$ years old. 
Table 1 Classification of health behaviors and metabolic risk factors

\begin{tabular}{llll}
\hline Variables & Ideal & Intermediate & Poor \\
\hline $\begin{array}{l}\text { Smoking status } \\
\begin{array}{l}\text { Alcohol } \\
\text { consumption }\end{array}\end{array}$ & Non-smoker & Previous smoker (smoking cessation $\geq 1$ year) & Current smoker \\
$\begin{array}{l}\text { Sleep duration, } \\
\text { hours }\end{array}$ & $7 \sim 8$ & Occasional drinker & $\begin{array}{l}\text { Regular drinker (weekly or monthly } \\
\text { drinking) }\end{array}$ \\
BMI, $\mathbf{k g} / \mathbf{m}^{2}$ & $5-6$ or $9-10$ & $<5$ or $>10$ \\
WHR $($ Male) & $<24$ & $24-27.9$ & $\geq 28$ \\
(Female) & $<0.90$ & $0.90-0.94$ & $\geq 0.95$ \\
BP, $\mathbf{m m H g}$ & $<0.85$ & $0.85-0.89$ & $\geq 0.90$ \\
& Non-drug therapy $<120 /$ & $120-139 / 80-89$ or $<140 / 90$ if taking an & $\geq 140 / 90$ \\
FBG, $\mathbf{m m o l} / \mathbf{L}$ & Nontihypertensive & \\
PA, (MET-hour/day) & $\geq$ P50 & $5.6-6.9$ or $<5.6$ if taking diabetes drugs & $\geq 7.0$ \\
\hline
\end{tabular}

$B M I$ Body mass index, FBG Fasting blood glucose, WHR Waist-to-hip ratio, MET Metabolic equivalent task, BP Blood pressure, PA Physical activity

All statistical analysis were performed using IBM SPSS statistics for windows, version 22.0 (IBM Corp., Armonk, NY, USA) and R 4.0.2 (R Core Team). Statistical tests were two-tailed and statistical significance was set at $P \leq 0.05$.

\section{Results}

\section{Characteristics of participants}

Of the 5559 participants entering this study, 5032 (90.5\%) completed questionnaire and physical examination. Women comprised $63.3 \%$ of the sample $(n=$ 3187). Participants were excluded if they had missing data on those variables fasting time $<8 \mathrm{~h}(n=8)$, lacked of blood samples $(n=483)$, or were using hyperlipidemic medication $(n=36)$.

The demographic characteristics are presented in Table 2. The average age of the participants was $(51.8 \pm$ 11.7) years. Men were generally older, had more education compared to women. Most men worked as farmers while women worked in other occupations.

\section{Distribution of health behaviors and metabolic risk factors}

Table 3 summarizes the distribution of health behaviors and metabolic risk factors by gender, age and area of residence. Overall, the proportion of subjects with ideal WHR (32.9\%), BMI (40.2\%) and BP (33.4\%) were relatively low. However, there were high percentages of both non-smokers (78.1\%) and of those with ideal FBG (73.5\%) levels. Current smokers and regular drinkers were more likely to be men, to be older than 60 , and living in rural areas. About half of all women had a poor WHR (50.2\%). But among women, nearly all were nonsmokers (98.9\%), a majority were non-drinkers (53.7\%), and over three-quarter had ideal FBG (77.7\%).

\section{Prevalence of dyslipidemia}

The prevalence of dyslipidemia in this cohort of Miao participants was $32.8 \%$, which was age-standardized was 23.3\% (Table 4). Dyslipidemia was more likely to be present in men than women $(42.2 \%$ vs $27.5 \%, P<0.001)$. In both genders, there was more dyslipidemia in participants who had poor WHR, BMI, FBG, and BP (each $P<0.001$ ). Table 5 presents the prevalence of dyslipidemia components. The prevalence of high TC, TG, LDL-C and low HDL-C was 10.4\%, 21.8\%, 7.7\% and $7.4 \%$, respectively. Again, participants with poor WHR, $\mathrm{BMI}, \mathrm{FBG}$ and BP were more likely to have prevalence of dyslipidemia components.

\section{Association of health behaviors and metabolic risk factors with dyslipidemia}

Figures 1 and 2 present the unadjusted and adjusted association of health behaviors and metabolic risk factors with dyslipidemia by gender, respectively. Figure 2 adjusted for age, residence, education, occupation, marital status and family income. High TG is likely to appear in subjects with poor BMI, WHR, BP and FBG. The odds of finding high lipid levels in subjects with poor WHR were high. The strongest associations were in subjects with poor WHR and high LDL-C (adjusted OR $=3.09$ for men and adjusted $\mathrm{OR}=3.20$ for women, $P<0.001$ ), low HDL-C (adjusted OR $=2.39$ for men and adjusted $\mathrm{OR}=3.04$ for women, $P<0.001)$. The odds of having low HDL-C was the highest in poor FBG group in women (adjusted $\mathrm{OR}=2.71$, 95\% CI: 1.43-5.14). Men who reported drinking regularly were more inclined to also have high TG (adjusted OR $=1.61$, 95\% CI: $1.19-$ 2.20 ), but were less likely to have high LDL-C (adjusted $\mathrm{OR}=0.51,95 \%$ CI: 0.32-0.81). Males with current smoking were an independent risk factor only for low HDL-C (adjusted OR $=1.44,95 \% \mathrm{CI}: 1.05-1.99$ ). 
Table 2 Characteristics of participants

\begin{tabular}{|c|c|c|c|c|}
\hline Variables & Total $(n=5032)$ & Male $(n=1845)$ & Female $(n=3187)$ & $P$ - value ${ }^{*}$ \\
\hline Age (years, \%) & & & & $<0.001$ \\
\hline $30-39$ & $862(17.2)$ & $277(15.0)$ & $585(18.4)$ & \\
\hline $40-49$ & $1478(29.4)$ & $500(27.1)$ & $978(30.6)$ & \\
\hline $50-59$ & $1396(27.7)$ & $490(26.6)$ & $906(28.4)$ & \\
\hline $60-69$ & $893(17.7)$ & $397(21.5)$ & $496(15.6)$ & \\
\hline 70-79 & $403(8.0)$ & $181(9.8)$ & $222(7.0)$ & \\
\hline Area of residence (\%) & & & & 0.056 \\
\hline Rural & $3584(71.2)$ & $1289(69.9)$ & $2295(72.0)$ & \\
\hline Urban & $1448(28.8)$ & $556(30.1)$ & $892(28.0)$ & \\
\hline Educational level (\%) & & & & $<0.001$ \\
\hline No formal school & $2190(43.5)$ & $540(29.2)$ & $1650(51.7)$ & \\
\hline Primary school & $687(13.7)$ & $330(17.9)$ & $357(11.2)$ & \\
\hline Middle school & $1004(20.0)$ & $400(21.7)$ & $604(19.0)$ & \\
\hline High school or college & $884(17.5)$ & $418(22.7)$ & $466(14.6)$ & \\
\hline University or above & $267(5.3)$ & $157(8.5)$ & $110(3.5)$ & \\
\hline Occupation (\%) & & & & $<0.001$ \\
\hline Farmers & $1767(35.1)$ & $725(39.3)$ & $1042(32.7)$ & \\
\hline Workers & $400(7.9)$ & $186(10.1)$ & $214(6.7)$ & \\
\hline Administrators & $165(3.3)$ & $95(5.1)$ & $70(2.2)$ & \\
\hline Specialists & $441(8.8)$ & $237(12.8)$ & $204(6.4)$ & \\
\hline Other occupations & 2259 (44.9) & $602(32.7)$ & $1657(52.0)$ & \\
\hline Marital status (\%) & & & & $<0.001$ \\
\hline Married or cohabiting & $4367(86.8)$ & $1644(89.1)$ & $2723(85.4)$ & \\
\hline Separated or divorced & $183(3.6)$ & $62(3.4)$ & $121(3.8)$ & \\
\hline Widowed & $427(8.5)$ & $87(4.7)$ & $340(10.7)$ & \\
\hline Never married & $55(1.1)$ & $52(2.8)$ & $3(0.1)$ & \\
\hline \multicolumn{5}{|c|}{ Family income (yuan/year, \%) } \\
\hline$<20,000$ & $2101(41.7)$ & $772(41.8)$ & $1329(41.7)$ & 0.783 \\
\hline $20,000-59,999$ & $1650(32.8)$ & $591(32.0)$ & $1059(33.2)$ & \\
\hline $60,000-99,999$ & $763(15.2)$ & $289(15.7)$ & $474(14.9)$ & \\
\hline$\geq 100,000$ & $518(10.3)$ & $193(10.5)$ & $325(10.2)$ & \\
\hline
\end{tabular}

Data was presented as $\mathrm{n}(\%)$

${ }^{*}$ Chi-square test

Figures 3 and 4, respectively, summarizes the unadjusted and adjusted association of health behaviors and metabolic risk factors with dyslipidemia by age. Stratification by age did not change the association between high TG and poor BMI, WHR, BP and FBG. In participants $\geq 60$ years old, there was a strong association between poor WHR ang elevated LDL-C (adjusted OR = 5.24,95\%CI: 2.42-11.34). Poor BP was connected to the elevated TC in older subjects (adjusted $\mathrm{OR}=2.48$, 95\%CI:1.31-4.70). These associations were also strong in subjects < 60 years old. Subjects who reported current and past smoking were associated reduced HDL-C levels without regard to age (age group < 60 years: adjusted
OR $=2.79,95 \%$ CI: $2.05-3.80$; age group $\geq 60$ years: adjusted OR $=2.96,95 \% \mathrm{CI}: 1.67-5.25)$.

\section{Discussion}

This study summarizes the epidemiological characteristics of one minority group within the large-scale CMEC study in China. We found clear evidence that several major health behaviors and metabolic risk factors were associated with dyslipidemia. This was particularly true for metabolic risk factors. These findings have considerable practical relevance for the prevention of dyslipidemia in ethnic minority groups. These factors have been identified as important bio-markers for the presence of 
Table 3 Distribution of health behaviors and metabolic risk factors by gender, age and area of residence

\begin{tabular}{|c|c|c|c|c|c|c|c|}
\hline \multirow[t]{2}{*}{ Variables } & \multirow[t]{2}{*}{ Total } & \multicolumn{2}{|l|}{ Gender } & \multicolumn{2}{|l|}{ Age (years) } & \multicolumn{2}{|l|}{ Area of residence } \\
\hline & & Male $(n=1845)$ & Female $(n=3187)$ & $<60(n=3736)$ & $\geq 60(n=1296)$ & Rural $(n=3584)$ & Urban $(n=1448)$ \\
\hline \multicolumn{8}{|c|}{ Smoking status (\%) } \\
\hline Ideal & $3933(78.1)$ & $780(42.1)$ & $3153(98.9)$ & $2964(79.3)$ & $969(74.8)$ & $2799(78.1)$ & $1134(78.3)$ \\
\hline Intermediate & $190(3.8)$ & $184(10.1)$ & $6(0.2)$ & $121(3.2)$ & $69(5.3)$ & $122(3.4)$ & $68(4.7)$ \\
\hline Poor & $909(18.1)$ & $881(47.8)$ & $28(0.9)$ & $651(17.5)$ & $258(19.9)$ & $663(18.5)$ & $246(17.0)$ \\
\hline \multicolumn{8}{|c|}{ Alcohol consumption (\%) } \\
\hline Ideal & $2251(44.7)$ & $541(29.3)$ & $1710(53.7)$ & $1514(40.6)$ & $737(56.9)$ & $1662(46.4)$ & $589(40.7)$ \\
\hline Intermediate & $1881(37.4)$ & $709(38.5)$ & $1172(36.7)$ & $1559(41.7)$ & $322(24.8)$ & $1273(35.5)$ & $608(42.0)$ \\
\hline Poor & $900(17.9)$ & $595(32.2)$ & 305 (9.6) & $663(17.7)$ & $237(18.3)$ & $649(18.1)$ & $251(17.3)$ \\
\hline \multicolumn{8}{|c|}{ PA (MET- hour/day, \%) } \\
\hline Ideal & $2516(50.0)$ & $948(51.4)$ & $1568(49.2)$ & $2056(55.0)$ & $460(35.5)$ & $1985(55.4)$ & $531(36.7)$ \\
\hline Intermediate & $1258(25.0)$ & $445(24.1)$ & $813(25.5)$ & $926(24.8)$ & $332(25.6)$ & 809 (22.6) & $449(31.0)$ \\
\hline Poor & $1258(25.0)$ & $452(24.5)$ & $806(25.3)$ & $754(20.2)$ & $504(38.9)$ & $790(22.0)$ & $468(32.3)$ \\
\hline \multicolumn{8}{|l|}{ WHR (\%) } \\
\hline Ideal & $1654(32.9)$ & $788(42.7)$ & $866(27.1)$ & $1267(33.9)$ & $387(29.8)$ & 1197 (33.4) & 457 (31.6) \\
\hline Intermediate & $1184(23.5)$ & $462(25.1)$ & $722(22.7)$ & $906(24.3)$ & $278(21.5)$ & $812(22.7)$ & $372(25.7)$ \\
\hline Poor & 2194 (43.6) & $595(32.2)$ & $1599(50.2)$ & $1563(41.8)$ & $631(48.7)$ & 1575 (43.9) & $619(42.7)$ \\
\hline \multicolumn{8}{|l|}{ BMI $\left(\mathrm{kg} / \mathrm{m}^{2}, \%\right)$} \\
\hline Ideal & $2021(40.2)$ & $782(42.4)$ & 1239 (38.9) & $1429(38.2)$ & $592(45.7)$ & $1482(41.4)$ & $539(37.2)$ \\
\hline Intermediate & $2093(41.6)$ & $776(42.0)$ & $1317(41.3)$ & $1605(43.0)$ & $488(37.6)$ & 1449 (40.4) & $644(44.5)$ \\
\hline Poor & $918(18.2)$ & $287(15.6)$ & $631(19.8)$ & $702(18.8)$ & $216(16.7)$ & $653(18.2)$ & $265(18.3)$ \\
\hline \multicolumn{8}{|l|}{ FBG (mmol/L, \%) } \\
\hline Ideal & 3701 (73.5) & $1226(66.5)$ & $2475(77.7)$ & $2843(76.1)$ & $858(66.2)$ & $2704(75.4)$ & $997(68.9)$ \\
\hline Intermediate & 1035 (20.6) & $458(24.8)$ & $577(18.1)$ & $714(19.1)$ & $321(24.8)$ & $706(19.7)$ & $329(22.7)$ \\
\hline Poor & $296(5.9)$ & $161(8.7)$ & $135(4.2)$ & $179(4.8)$ & $117(9.0)$ & $174(4.9)$ & $122(8.4)$ \\
\hline \multicolumn{8}{|l|}{ BP (mmHg, \%) } \\
\hline Ideal & $1683(33.4)$ & $391(21.2)$ & $1292(40.5)$ & $1473(39.4)$ & $210(16.2)$ & $1200(33.5)$ & $483(33.3)$ \\
\hline Intermediate & $1862(37.0)$ & $741(40.2)$ & $1121(35.2)$ & $1347(36.1)$ & $515(39.7)$ & $1331(37.1)$ & $531(36.7)$ \\
\hline Poor & 1478 (29.6) & $713(38.6)$ & $774(24.3)$ & $916(24.5)$ & $571(44.1)$ & $1053(29.4)$ & $434(30.0)$ \\
\hline \multicolumn{8}{|c|}{ Sleep duration (hour, \%) } \\
\hline Ideal & $2579(51.3)$ & $904(49.0)$ & $1675(52.6)$ & $2041(54.6)$ & $538(41.5)$ & $298(8.3)$ & $75(5.2)$ \\
\hline Intermediate & $2080(41.3)$ & $776(42.1)$ & $1304(40.9)$ & $1505(40.3)$ & $575(44.4)$ & $1540(43.0)$ & $540(37.3)$ \\
\hline Poor & $373(7.4)$ & 165 (8.9) & $208(6.5)$ & $190(5.1)$ & $183(14.1)$ & $1746(48.7)$ & 833 (57.5) \\
\hline
\end{tabular}

Data was presented as $\mathrm{n}(\%)$

$B M I$ Body mass index, FBG Fasting blood glucose, WHR Waist-to-hip ratio, BP Blood pressure, PA Physical activity, MET Metabolic equivalent task

dyslipidemia and are also associated with atherosclerotic diseases [18]. Notably, these health behaviors and metabolic factors associated with dyslipidemia can be largely controlled by adopting healthy lifestyle. Dyslipidemia is a part of a larger metabolic syndrome (MS) that puts people at risk for CVD and death, which has long been demonstrated in Framingham and other studies [19, 20]. Therefore, a prevention strategy can be formulated to reduce the prevalence of dyslipidemia, which will then serve to depress CVD.
This is the first study to systematically evaluated the prevalence of dyslipidemia among the ethnic Miao adult population of southwest China. Our analysis describes a dyslipidemia prevalence of $32.8 \%$, which is lower than the national average of $40.4 \%$ [17], was also slightly lower than that reported in Guangxi, Jiangsu, Beijing [21-23]. Although formal comparisons have not been made, differences between populations may be related to genetic predisposition, and socioeconomic conditions of the participants $[8,24]$. In addition, the prevalence of dyslipidemia components were significantly different. 
Table 4 Adjusted odds ratio $(95 \% \mathrm{Cl})$ of dyslipidemia prevalence

\begin{tabular}{|c|c|c|c|c|}
\hline \multirow[t]{2}{*}{ Variables } & \multicolumn{2}{|l|}{ Male $(n=778)$} & \multicolumn{2}{|c|}{ Female $(n=875)$} \\
\hline & Dyslipidemia & OR $(95 \% C l)^{a}$ & Dyslipidemia & OR $(95 \% C l)^{a}$ \\
\hline \multicolumn{5}{|c|}{ Smoking status (\%) } \\
\hline Ideal & $326(41.8)$ & 1.00 & $869(27.6)$ & 1.00 \\
\hline Intermediate & $73(39.7)$ & $0.64(0.70-5.86)$ & $1(16.7)$ & $0.73(0.08-6.65)$ \\
\hline Poor & $379(43.0)$ & $0.67(0.24-1.83)$ & $5(17.9)$ & $0.69(0.25-1.94)$ \\
\hline \multicolumn{5}{|c|}{ Alcohol consumption (\%) } \\
\hline Ideal & $190(35.1)$ & 1.00 & $498(29.1)$ & 1.00 \\
\hline Intermediate & $331(46.7)$ & $0.90(0.75-1.07)$ & $290(24.7)$ & $0.89(0.74-1.07)$ \\
\hline Poor & $257(43.2)$ & $1.07(0.81-1.43)$ & $87(28.5)$ & $1.09(0.81-1.45)$ \\
\hline \multicolumn{5}{|c|}{ PA (MET-hour/day, \%) } \\
\hline Ideal & $393(41.5)$ & 1.00 & $380(24.2)$ & 1.00 \\
\hline Intermediate & $202(45.4)$ & $1.23(1.00-1.50)$ & $234(28.8)$ & $1.21(0.98-1.49)$ \\
\hline Poor & $183(40.5)$ & $1.27(1.04-1.55)^{*}$ & $261(32.4)$ & $1.26(1.02-1.57)^{*}$ \\
\hline \multicolumn{5}{|l|}{ WHR (\%) } \\
\hline Ideal & $218(27.7)$ & 1.00 & $132(15.2)$ & 1.00 \\
\hline Intermediate & $208(45.0)$ & $1.33(1.02-1.72)^{*}$ & $172(23.8)$ & $1.28(0.98-1.67)$ \\
\hline Poor & $352(59.2)$ & $1.92(1.52-2.42)^{*}$ & $571(35.7)$ & $1.83(1.44-2.33)^{*}$ \\
\hline \multicolumn{5}{|l|}{ BMI $\left(\mathrm{kg} / \mathrm{m}^{2}, \%\right)$} \\
\hline Ideal & $205(26.2)$ & 1.00 & $226(18.2)$ & 1.00 \\
\hline Intermediate & $400(51.5)$ & $1.49(1.22-1.82)^{*}$ & $396(30.1)$ & $1.49(1.22-1.83)^{*}$ \\
\hline Poor & $173(60.3)$ & $1.85(1.46-2.35)^{*}$ & $253(40.1)$ & $1.87(1.47-2.37)^{*}$ \\
\hline \multicolumn{5}{|l|}{ FBG (mmol/L, \%) } \\
\hline Ideal & 449 (36.6) & 1.00 & $576(23.3)$ & 1.00 \\
\hline Intermediate & $223(48.7)$ & $1.53(1.25-1.87)^{*}$ & $218(37.8)$ & $1.50(1.22-1.85)^{*}$ \\
\hline Poor & $106(65.8)$ & $3.16(2.18-4.58)^{*}$ & $81(60.0)$ & $3.08(2.12-4.48)^{*}$ \\
\hline \multicolumn{5}{|l|}{ BP (mmHg, \%) } \\
\hline Ideal & $122(31.2)$ & 1.00 & $238(18.4)$ & 1.00 \\
\hline Intermediate & $308(41.6)$ & $1.49(1.22-1.81)^{*}$ & $338(30.2)$ & $1.45(1.18-1.78)^{*}$ \\
\hline Poor & $348(48.8)$ & $1.87(1.50-2.32)^{*}$ & $299(38.6)$ & $1.81(1.44-2.28)^{*}$ \\
\hline \multicolumn{5}{|c|}{ Sleep duration (hour, \%) } \\
\hline Ideal & 379 (41.9) & 1.00 & $444(26.5)$ & 1.00 \\
\hline Intermediate & $338(43.6)$ & $0.93(0.79-1.11)$ & $359(27.5)$ & $0.94(0.79-1.12)$ \\
\hline Poor & $61(37.0)$ & $1.08(0.78-1.49)$ & $72(34.6)$ & $1.11(0.79-1.54)$ \\
\hline
\end{tabular}

BMI Body mass index, FBG Fasting blood glucose, WHR Waist-to-hip ratio, BP Blood pressure, PA Physical activity, OR Odds ratio, $C I$ Confidence interval, MET Metabolic equivalent task

${ }^{*} P<0.001$

${ }^{a}$ Adjusted for demographic characteristics, including age, area of residence, education, occupation, marital status and family income

The most common component of dyslipidemia was elevated TG, with prevalence of $21.8 \%$ and was consistent with other studies $[25,26]$. This phenomenon may reflects the increasing popularity of carbohydrates and high cholesterol diets in recent decades [27].

We found a consistent pattern of increasing likelihood for the presence of dyslipidemia with other indicators of poor cardiovascular health: poor WHR, BMI, BP and FBG. The prevalence of metabolic risk factors are indicative that dyslipidemia is likely to be present. In light of these results, screening for dyslipidemia should be one of the monitoring interventions conducted in the Miao people when obesity, hypertension, or hyperglycemia is observed.

Regardless of age or gender, poor BMI, WHR, FBG and $\mathrm{BP}$ results were commonly associated with elevated TG, which corresponds with the results of previous studies [28, 29]. Again, if one metabolic risk factor is 
Table 5 Adjusted odds ratio $(95 \% \mathrm{Cl})$ of dyslipidemia components prevalence

\begin{tabular}{|c|c|c|c|c|c|c|c|c|}
\hline \multirow[t]{2}{*}{ Variables } & \multicolumn{2}{|c|}{$\mathrm{TC}(\geq 6.22 \mathrm{mmol} / \mathrm{L})$} & \multicolumn{2}{|c|}{$\mathrm{TG}(\geq 2.26 \mathrm{mmol} / \mathrm{L})$} & \multicolumn{2}{|c|}{ LDL-C ( $\geq 4.14 \mathrm{mmol} / \mathrm{L})$} & \multicolumn{2}{|c|}{$\mathrm{HDL}-\mathrm{C}(<1.04 \mathrm{mmol} / \mathrm{L})$} \\
\hline & n (\%) & OR $(95 \% \mathrm{Cl})$ & n (\%) & OR $(95 \% \mathrm{Cl})$ & n (\%) & OR $(95 \% \mathrm{Cl})$ & n (\%) & OR $(95 \% \mathrm{Cl})$ \\
\hline \multicolumn{9}{|c|}{ Smoking status (\%) } \\
\hline Ideal & $417(10.6)$ & 1.00 & $771(19.6)$ & 1.00 & $304(7.7)$ & 1.00 & $227(5.8)$ & 1.00 \\
\hline Intermediate & $23(12.1)$ & $1.08(0.68-1.72)$ & $52(27.4)$ & $1.60(1.12-2.30)^{\#}$ & $12(6.3)$ & $0.78(0.42-1.45)$ & $20(10.5)$ & $2.30(1.38-3.81)^{\#}$ \\
\hline Poor & $94(10.3)$ & $0.96(0.74-1.25)$ & $275(30.3)$ & $1.70(1.40-2.06)^{*}$ & $69(7.6)$ & $1.15(0.84-1.55)$ & $124(13.6)$ & $2.86(2.19-3.73)^{*}$ \\
\hline \multicolumn{9}{|c|}{ Alcohol consumption (\%) } \\
\hline Ideal & $248(11.0)$ & 1.00 & $412(18.3)$ & 1.00 & $191(8.5)$ & 1.00 & $143(6.4)$ & 1.00 \\
\hline Intermediate & $191(10.2)$ & $1.02(0.83-1.27)$ & $430(22.9)$ & $1.18(0.99-1.39)$ & $131(7.0)$ & $0.82(0.64-1.04)$ & $143(7.6)$ & $1.01(0.78-1.30)$ \\
\hline Poor & 95 (10.6) & $0.93(0.71-1.22)$ & $256(28.4)$ & $1.34(1.09-1.65)^{\#}$ & $63(7.0)$ & $0.75(0.54-1.04)$ & $85(9.4)$ & $1.02(0.74-1.40)$ \\
\hline \multicolumn{9}{|c|}{ PA (MET-hour/day, \%) } \\
\hline Ideal & $241(9.6)$ & 1.00 & $513(20.4)$ & 1.00 & $172(6.8)$ & 1.00 & $156(6.2)$ & 1.00 \\
\hline Intermediate & $136(10.8)$ & $1.03(0.81-1.30)$ & $302(24.0)$ & $1.13(0.94-1.37)$ & $99(7.9)$ & $0.96(0.73-1.27)$ & $115(9.1)$ & $1.45(1.11-1.89)^{*}$ \\
\hline Poor & $157(12.5)$ & $1.11(0.87-1.41)$ & $283(22.5)$ & $1.17(0.98-1.40)$ & $114(9.1)$ & $0.97(0.74-1.29)$ & $100(7.9)$ & $1.31(0.98-1.76)$ \\
\hline \multicolumn{9}{|l|}{ WHR (\%) } \\
\hline Ideal & $110(6.7)$ & 1.00 & $196(11.9)$ & 1.00 & $58(3.5)$ & 1.00 & $68(4.1)$ & 1.00 \\
\hline Intermediate & $107(9.0)$ & $1.07(0.80-1.44)$ & $270(22.8)$ & $1.63(1.31-2.03)^{*}$ & $76(6.4)$ & $1.62(1.13-2.33)^{\#}$ & $74(6.3)$ & $1.38(0.97-1.98)$ \\
\hline Poor & $317(14.4)$ & $1.58(1.21-2.05)^{*}$ & $632(28.8)$ & $1.98(1.61-2.43)^{*}$ & $251(11.4)$ & $2.81(2.02-3.90)^{*}$ & $229(10.4)$ & $2.32(1.68-3.20)^{*}$ \\
\hline \multicolumn{9}{|l|}{ BMI $\left(\mathrm{kg} / \mathrm{m}^{2}, \%\right)$} \\
\hline Ideal & $160(7.9)$ & 1.00 & $230(11.4)$ & 1.00 & $115(5.7)$ & 1.00 & $82(4.1)$ & 1.00 \\
\hline Intermediate & $242(11.6)$ & $1.13(0.89-1.42)$ & $550(26.3)$ & $1.99(1.65-2.39)^{*}$ & $174(8.3)$ & $1.00(0.77-1.30)$ & $185(8.8)$ & $1.81(1.36-2.43)^{*}$ \\
\hline Poor & $132(14.4)$ & $1.18(0.89-1.55)$ & $318(34.6)$ & $2.48(1.99-3.10)^{*}$ & $96(10.5)$ & $1.01(0.74-1.39)$ & $104(11.3)$ & $2.10(1.49-2.95)^{*}$ \\
\hline \multicolumn{9}{|l|}{ FBG (mmol/L, \%) } \\
\hline Ideal & $321(8.7)$ & 1.00 & $645(17.4)$ & 1.00 & $234(6.3)$ & 1.00 & $224(6.1)$ & 1.00 \\
\hline Intermediate & $144(13.9)$ & $1.30(1.04-1.62)^{\#}$ & $308(29.8)$ & $1.55(1.31-1.84)^{*}$ & $112(10.8)$ & $1.37(1.07-1.72)^{\#}$ & $99(9.6)$ & $1.32(1.01-1.72)^{\#}$ \\
\hline Poor & $69(23.3)$ & $2.08(1.53-2.84)^{*}$ & $145(49.0)$ & $2.97(2.27-3.88)^{*}$ & $39(13.2)$ & $1.39(1.00-2.04)^{\#}$ & $48(16.2)$ & $2.05(1.42-2.97)^{*}$ \\
\hline \multicolumn{9}{|l|}{ BP $(\mathrm{mmHg}, \%)$} \\
\hline Ideal & $96(5.7)$ & 1.00 & $210(12.5)$ & 1.00 & $84(5.0)$ & 1.00 & $92(5.5)$ & 1.00 \\
\hline Intermediate & $205(11.0)$ & $1.64(1.26-2.12)^{*}$ & $424(22.8)$ & $1.67(1.37-2.02)^{*}$ & $150(8.1)$ & $1.42(1.06-1.90)^{\#}$ & $153(8.2)$ & $1.25(0.94-1.66)$ \\
\hline Poor & $233(15.7)$ & $2.12(1.61-2.78)^{*}$ & $464(31.2)$ & $2.21(1.80-2.72)^{*}$ & $151(10.2)$ & $1.58(1.16-2.15)^{\#}$ & $126(8.5)$ & $1.07(0.77-1.47)$ \\
\hline \multicolumn{9}{|c|}{ Sleep duration (hour, \%) } \\
\hline Ideal & $267(10.4)$ & 1.00 & $544(21.1)$ & 1.00 & $189(7.3)$ & 1.00 & $179(6.9)$ & 1.00 \\
\hline Intermediate & $220(10.6)$ & $0.92(0.76-1.12)$ & $464(22.3)$ & $1.07(0.92-1.25)$ & $153(7.4)$ & $0.95(0.75-1.19)$ & $165(7.9)$ & $1.15(0.92-1.45)$ \\
\hline Poor & $47(12.6)$ & $1.00(0.71-1.41)$ & $90(24.1)$ & $1.22(0.92-1.62)$ & $43(11.5)$ & $1.49(0.98-2.15)$ & $27(7.2)$ & $1.05(0.67-1.63)$ \\
\hline
\end{tabular}

Ideal group was used as reference, and non-dyslipidemia as control group. Adjusted for age, area of residence, education, occupation, marital status and family income

BMI Body mass index, FBG Fasting blood glucose, WHR Waist-to-hip ratio, BP Blood pressure, PA Physical activity, MET Metabolic equivalent task, TC Total cholesterol, TG Triglyceride, LDL-C Low-density lipoprotein cholesterol, HDL-C High-density lipoprotein cholesterol, OR Odds ratio, Cl Confidence interval ${ }^{*} P<0.001, " * P<0.05$

detected, other risk factors such as TG should also monitored. Of note, poor WHR was positively associated with TC as well as other lipid components, regardless of gender and age. There is evidence that indicates WHR may well reflect body fat distribution, and the intracentral deposition of adipose tissue is connected to dyslipidemia [30, 31]. In Miao women, FBG is closely associated with elevated TC, LDL-C, TG, and low HDL-C. Recent evidence suggested that lipid levels may possess the ability to stimulate insulin release, which may contribute to higher FBG levels [32].

The present study found that Miao men smoke more than women. Males who are current smoking more likely to have low HDL-C than non-smokers. However, regardless of age stratification, current smokers were more likely to have elevated TG and low HDL-C. These findings were consistent with numerous previous studies and appears to be common across different populations 












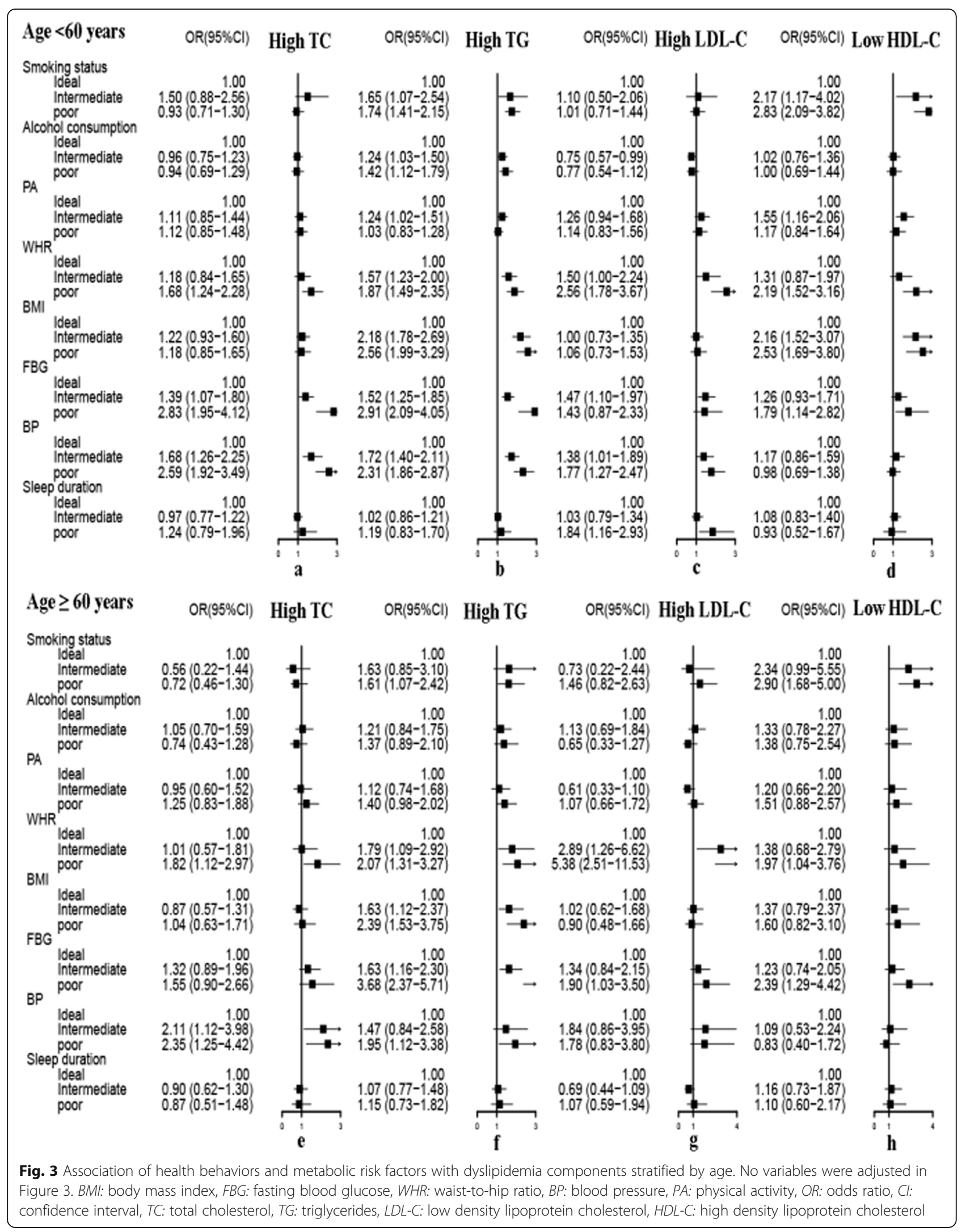




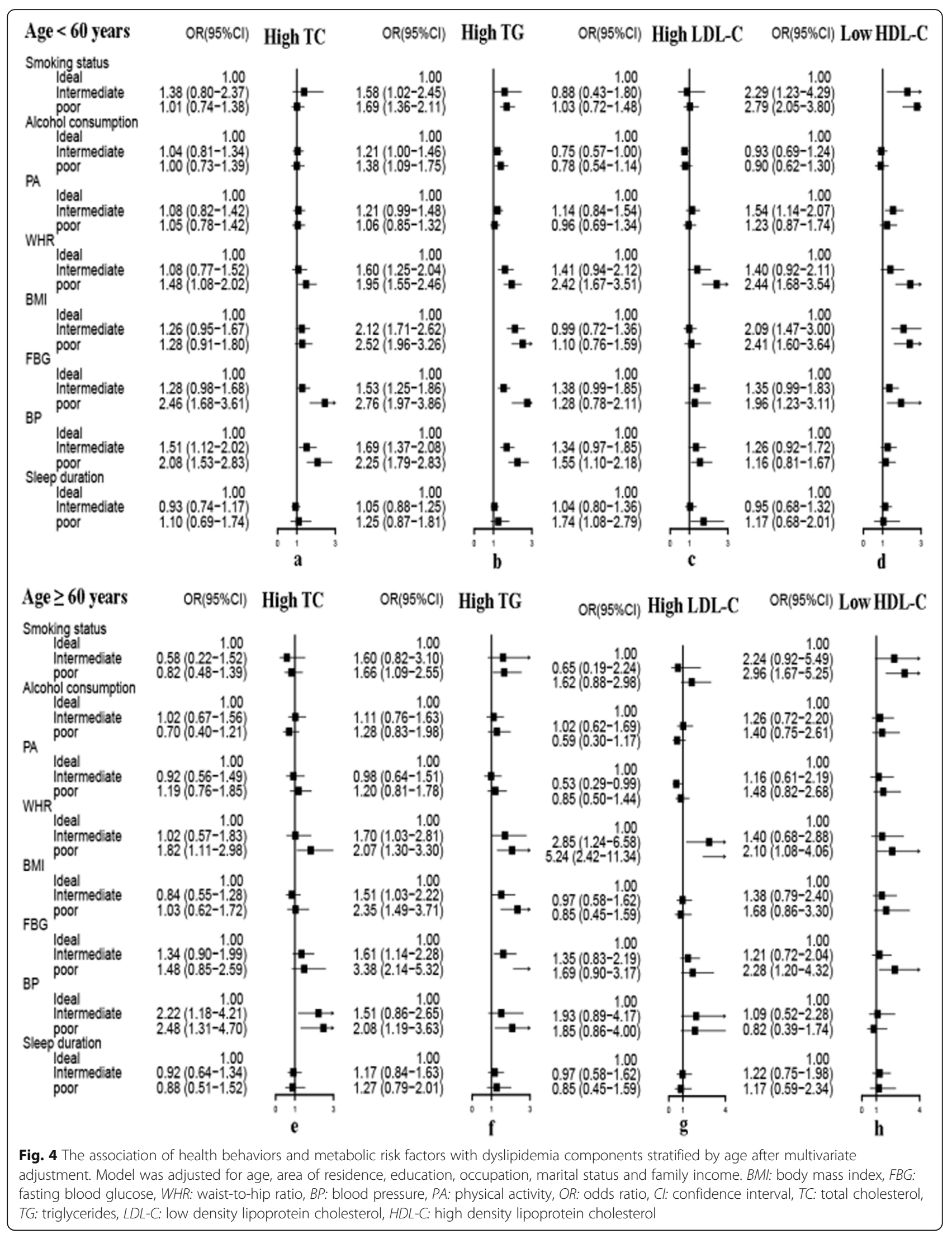


[33, 34]. Prior observations reported that smokers have increased TG and decreased the HDL-C, compared with non-smokers [35]. The impact of smoking on serum lipid levels is also dose-dependent, which may be the core mechanism by which smoking causes atherosclerosis [36]. Furthermore, our study found that drinking alcohol regularly was associated with elevated TG specifically in Miao men, or in subjects younger than 60 years old. Interestingly, this study also revealed that men who are regular drinkers were less likely to have elevated LDL-C than non-drinkers, which was different from the result of the Sun et al. [6] There is evidence to suggest that alcohol consumption can adversely affect the lipid levels. For example, wakabayashi et al. found that heavy drinkers showed detrimental effects on the TG and HDL [37]. A recent study reported that large amounts of alcohol consumption usually increased TG levels. The presence of drinking is a notable issue in atherosclerosis prevention and it might be more important for people with dyslipidemia to avoid alcohol consumption to depress the risk of CVD.

Other researchers observed that the risk of dyslipidemia is increased when physical activity is decreased [38]. However, the current study did not observed a significantly association between physical activity and dyslipidemia. Calculating the metabolic equivalent task of total physical activity based on subject reporting may not be the most precise way to estimate energy use. The present study did not distinguish domain-specific physical activity, thus a future study may more accurately assess the association between these two factors. Shigeki Kinuhata et al. observed a positive correlation between sleep duration and TG abnormality [39]. Zhan et al. also found that sleep duration was significantly associated with dyslipidemia in women, but not in men [40]. However, there was no association of sleep duration with any lipid components in current study. The present analysis focused on night-time sleep rather than on 24-h sleep, and data on sleep duration were collected from questionnaire, but not from objective measures. A metaanalysis also found that sleep duration was linearly associated with increased mortality risk [41]. Sleep quality may be an important factor in mechanisms linking sleep duration and health outcomes [42]. Therefore, further studies will be needed to investigate the association of sleep duration with health status.

Several limitations are present in this study. This analysis adjusted for factors that may confound the outcome, but residual confounding factors are possible. Behavioral factors such as smoking and alcohol use were self-reported, which may lead to misclassification. We conducted a cross-sectional study that cannot determine causation. Although we found that certain characteristics appeared together statistically, we could not determine which factor preceded another. A study using longitudinal follow-up data would be needed to assess the risk of an outcome. Nevertheless, this is the first known study in the Miao minority ethnic group to quantify behavioral, clinical, and metabolic factors and their relationship with dyslipidemia. This has considerable practical implications, findings from the present study have shed light on the cost-effective strategy for dyslipidemia management based on lifestyle changes in ethnic Chinese minority groups.

\section{Conclusions}

This large-scare study of Miao Chinese adults provided convincing evidence that several modifiable risk factors for CVD were closely linked with the prevalence of dyslipidemia. These findings may be used to inform the development of population-based CVD prevention strategies. Given the limited economic and medical conditions in regions with large ethnic minorities, such interventions are a cost-effective way to respond to the challenges posed by dyslipidemia and even CVD.

\section{Acknowledgements}

Particularly thanks to the Guiyang Center for Disease Control and Prevention, the affiliated hospital of Guizhou Medical University and local governments to assist and support the investigation work. Appreciation is expressed to the CMEC team members for conceiving and designing this study and their great contributions to initiate the project.

\section{Authors' contributions}

Each author has been involved in and contributed to this paper. F Nie carried out the statistical analysis and wrote the manuscript. F Hong and P Luo contributed to the study design of this paper. LW Du, ZY Wang, H Guan, JY Yang and QB Zeng participated in the data collection. JH Wang contributed to guide the paper and correcting the English. All authors read and approved the final manuscript.

\section{Funding}

This work was supported by the National key R\&D Program of china (N0.2017YFC0907301) and the Science and Technology Plan Project of Guizhou Province, China (NO.QKHPTRC[2018]5403). The funder played no role in the design of the study and collection, analysis, and interpretation of data and in writing the manuscript.

\section{Availability of data and materials}

Currently, the database used to support this study are not freely available in view of participants' privacy protection but are available from the corresponding author on reasonable data request. Researchers interested in our study could contact the corresponding author Dr. Feng Hong (51949096 7@qq.com) who will review the data request.

\section{Declarations}

\section{Ethics approval and consent to participate}

This study protocol was conducted in accordance with the principles of the Declaration of Helsinki and was approved by the Medical Ethical Committee of Sichuan University (K2016038) and the Ethics Committee of the Affiliated Hospital of Guizhou Medical University (2018[094]). And written informed consent was obtained from each subject. 


\section{Consent for publication}

Not Applicable.

\section{Competing interests}

The authors declare that they have no competing interests.

\section{Author details}

${ }^{1}$ School of Public Health, The Key Laboratory Of Environmental Pollution Monitoring and Disease Control, Ministry of Education, Guizhou Medical University, Guiyang 550025, China. ${ }^{2}$ School of Public Health, Guizhou Medical University, Guiyang 550025, China.

\section{Received: 17 November 2020 Accepted: 19 April 2021} Published online: 03 May 2021

\section{References}

1. Weiwei C, Runlin G, Lisheng L, Manlu Z, Wen W, Yongjun W, et al. Outline of the report on cardiovascular diseases in China, 2014. Eur Heart J Suppl. 2016;18(suppl F):F2-F11. https://doi.org/10.1093/eurheartj/suw030.

2. Yusuf S, Bosch J, Dagenais G. Cholesterol lowering in intermediate-risk persons without cardiovascular disease. J Vasc Surg. 2016;64(3):827. https:// doi.org/10.1016/j.jvs.2016.07.054.

3. Katzmann JL, Laufs U. New insights in the control of low-density lipoprotein cholesterol to prevent cardiovascular disease. Curr Cardiol Rep. 2019;21:1-9. https://doi.org/10.1007/s11886-019-1159-z.

4. Baigent C, Keech A, Kearney PM, Blackwell L, Buck G, Pollicino C, et al. Efficacy and safety of cholesterol-lowering treatment: prospective metaanalysis of data from 90,056 participants in 14 randomised trials of statins. Lancet. 2005;366:1267-78. https://doi.org/10.1016/50140-6736(05)67394-1.

5. Wang B, Wei D, Wang C, Zhang J, Pan L, Ma M, et al. Prevalence of dyslipidemia and associated factors in the Yi farmers and migrants of southwestern China. Atherosclerosis. 2012;223(2):512-8. https://doi.org/10.1016/j.atherosclerosis.2012. 06.009 .

6. Sun GZ, Li Z, Guo L, Zhou Y, Yang HM, Sun YX. High prevalence of dyslipidemia and associated risk factors among rural Chinese adults. Lipids Health Dis. 2014;13(1):189. https://doi.org/10.1186/1476-511X-13-189.

7. Dong Z. A review of epidemiological studies on blood lipids in Chinese population in the past 70 years. China Med. 2019;14:1441-4.

8. Qi L, Ding X, Tang W, Li Q, Mao D, Wang Y. Prevalence and risk factors associated with Dyslipidemia in Chongqing, China. Int J Environ Res Public Health. 2015:12(10):13455-65. https://doi.org/10.3390/ijerph121013455.

9. Chen $Q$, Hua $C$, Zhon B, Wang F, Xu X. Analysison prevalence of overweight and obesity and theirrelation with diabetes, hypertension, dyslipidemia among adults in Pinghu city. Shanghai J Prev Med. 2016;28:361-5. https:// doi.org/10.19428/j.cnki.sjpm.2016.06.003.

10. Zhao Y, Liu X, Mao Z, Hou J, Huo W, Wang C, et al. Relationship between multiple healthy lifestyles and serum lipids among adults in rural China: a population-based cross-sectional study. Prev Med. 2020;138:106158. https:// doi.org/10.1016/j.ypmed.2020.106158.

11. Dudum R, Juraschek SP, Appel LJ. Dose-dependent effects of lifestyle interventions on blood lipid levels: results from the PREMIER trial. Patient Educ Couns. 2019;102(10):1882-91. https://doi.org/10.1016/.pec.2019.05.005.

12. Zhang $X$. The impact of the migration of the Chinese Miao from their rural villages to cities. Guizhou Ethnic Stud. 2013;34:41-4.

13. Liu G, Mao L, Li N. Health values and health-related behaviors in ethnic minority groups in Guizhou province. J Sichuan Univ (Med Ed). 2007;38(3):475-9.

14. Zhao X, Hong F, Yin J, Tang W, Zhang G, Liang X, et al. Cohort profile: the China Multi-Ethnic Cohort (CMEC) study; 2020. https://doi.org/10.1101/2020. 02.14.20022970.

15. Zhu N, Yu C, Guo Y, Bian Z, Han Y, Yang L, et al. Adherence to a healthy lifestyle and all-cause and cause-specific mortality in Chinese adults: a 10year prospective study of 0.5 million people. Int J Behav Nutr Phys Act. 2019;16:98. https://doi.org/10.1186/s12966-019-0860-z.

16. Sacco RL. The new American Heart Association 2020 goal: achieving ideal cardiovascular health. J Cardiovasc Med. 2011;12(4):255-7. https://doi.org/1 0.2459/JCM.0b013e328343e986.

17. Dyslipidemia CJCO. Guidelines for prevention and treatment of dyslipidemia in Chinese adults (2016 revision). Chin J Circ. 2016:31:937-53.

18. Zaid M, Miura K, Okayama A, Nakagawa H, Sakata K, Saitoh S, et al. Associations of high-density lipoprotein particle and high-density lipoprotein cholesterol with alcohol intake, smoking, and body mass index- the INTERLIPID study. Circ J. 2018;82:2557-65. https://doi.org/10.1253/circj. CJ-18-0341.

19. O'Donnell CJ, Elosua R. Cardiovascular risk factors. Insights from Framingham heart study. Rev Esp Cardiol. 2008;61(3):299-310. https://doi. org/10.1157/13116658

20. Iqbal J, Al QA, Hawwari A, Alghanem AF, Ahmed G. Metabolic syndrome, dyslipidemia and regulation of lipoprotein metabolism. Curr Diabetes Rev. 2018;14(5):427-33. https://doi.org/10.2174/1573399813666170705161039.

21. Xi Y, Niu L, Cao N, Bao H, Xu X, Zhu H, et al. Prevalence of dyslipidemia and associated risk factors among adults aged $\geq 35$ years in northern China: a cross-sectional study. BMC Public Health. 2020;20(1):1068. https://doi.org/1 0.1186/s12889-020-09172-9.

22. Luo $\mathrm{S}$, Yang $\mathrm{H}$, Meng $\mathrm{X}$, Huang $\mathrm{T}, \mathrm{Xu} J, \mathrm{Xu}$ Y. Prevalence rate and risk factors of dyslipidemia among adults in Guangxi Province. Appl Prev Med. 2014;20:129-33.

23. Wang Y, Dai Y, Wang S, Zhang J, Zhu Q, Wei X. Prevalence and related factors of dyslipidemia among the adult residents in Jiangsu Province in 2014. J Hyg Res. 2019;48:945-52. https://doi.org/10.19813/j.cnki.weishengya njiu.2019.06.014.

24. Opoku S, Gan Y, Fu W, Chen D, Addo-Yobo E, Trofimovitch D, et al. Prevalence and risk factors for dyslipidemia among adults in rural and urban China: findings from the China National Stroke Screening and prevention project (CNSSPP). BMC Public Health. 2019;19. https://doi.org/10.1186/s12 889-019-7827-5.

25. Deng $Q$, Zhou X, Liang M, Yu H, Deng Q, Lu J, et al. Investigation on risk factors and differences of dyslipidemia between Mulam and Miao adult women; in Guangxi, China. Chin J Anat Clin. 2015:224-9. https://doi.org/10.3 760/cma.jissn.2095-7041.2015.03.010.

26. Li Z, Yang $R, X u G, X i a T$. Serum lipid concentrations and prevalence of dyslipidemia in a large professional population in Beijing. Clin Chem. 2005; 51:144-50. https://doi.org/10.1373/dlinchem.2004.038646.

27. Joshi SR, Anjana RM, Deepa M, Pradeepa R, Bhansali A, Dhandania VK, et al. Prevalence of dyslipidemia in urban and rural India: the ICMR-INDIAB study. PLoS One. 2014;9:e96808. https://doi.org/10.1371/journal.pone.0096808.

28. Bayram F, Kocer D, Gundogan K, Kaya A, Demir O, Coskun R, et al. Prevalence of dyslipidemia and associated risk factors in Turkish adults. J Clin Lipidol. 2014;8:206-16. https://doi.org/10.1016/j.jacl.2013.12.011.

29. Shen Z, Munker S, Wang C, Xu L, Ye H, Chen H, et al. Association between alcohol intake, overweight, and serum lipid levels and the risk analysis associated with the development of dyslipidemia. J Clin Lipidol. 2014;8(3): 273-8. https://doi.org/10.1016/j.jacl.2014.02.003.

30. Orces $\mathrm{CH}$, Montalvan M, Tettamanti D. Prevalence of abdominal obesity and its association with cardio metabolic risk factors among older adults in Ecuador. Diabetes Metab Syndr Clin Res Rev. 2017;11:S727-33. https://doi. org/10.1016/j.dsx.2017.05.006.

31. Klop B, Elte J, Cabezas M. Dyslipidemia in obesity: mechanisms and potential targets. Nutrients. 2013;5:1218-40. https://doi.org/10.3390/nu5041218.

32. Wang $L$, Tao $Y$, Xie Z, Ran $X$, Zhang $M$, Wang $Y$, et al. Prevalence of metabolic syndrome, insulin resistance, impaired fasting blood glucose, and dyslipidemia in Uygur and Kazak populations. I Clin Hypertens. 2010;12:7415. https://doi.org/10.1111/j.1751-7176.2010.00349.x.

33. Wannamethee G, Shaper AG. Blood lipids: the relationship with alcohol intake, smoking, and body weight. J Epidemiol Community Health. 1992;46: 197-202. https://doi.org/10.1136/jech.46.3.197.

34. Nakanishi N, Nakamura K, Ichikawa S, Suzuki K, Tatara K. Relationship between lifestyle and serum lipid and lipoprotein levels in middle-aged Japanese men. Eur J Epidemiol. 1999;15(4):341-8. https://doi.org/10.1023/a:1007527111946.

35. Moradinazar M, Pasdar Y, Najafi F, Shahsavari S, Shakiba E, Hamzeh B, et al. Association between dyslipidemia and blood lipids concentration with smoking habits in the Kurdish population of Iran. BMC Public Health. 2020; 20(1):673. https://doi.org/10.1186/s12889-020-08809-z.

36. Craig WY, Palomaki GE, Haddow JE. Cigarette smoking and serum lipid and lipoprotein concentrations: an analysis of published data. Br Med J. 1989; 298(6676):784-8. https://doi.org/10.1136/bmj.298.6676.784.

37. Wakabayashi I. Inverse association between excessive alcohol drinking and cardiometabolically healthy status in middle-aged men with and without overweight and obesity. Diabetes Metab Syndr. 2018;12(1):31-7. https://doi. org/10.1016/j.dsx.2017.08.009.

38. Zhou J, Zhou Q, Wang DP, Zhang T, Wang HJ, Song Y, et al. Associations of sedentary behavior and physical activity with dyslipidemia. Beijing Da Xue Xue Bao. 2017:49(3):418-23. https://doi.org/10.3969/j.issn.1671-167X.2016.03. 008. 
39. Kinuhata S, Hayashi T, Sato KK, Uehara S, Oue K, Endo G, et al. Sleep duration and the risk of future lipid profile abnormalities in middle-aged men: the Kansai healthcare study. Sleep Med. 2014;15(11):1379-85. https:// doi.org/10.1016/j.sleep.2014.06.011.

40. Zhan Y, Chen R, Yu J. Sleep duration and abnormal serum lipids: the China health and nutrition survey. Sleep Med. 2014;15(7):833-9. https://doi.org/1 0.1016/j.sleep.2014.02.006.

41. Jike M, Itani O, Watanabe N, Buysse DJ, Kaneita Y. Long sleep duration and health outcomes: a systematic review, meta-analysis and meta-regression. Sleep Med Rev. 2018;39:25-36. https://doi.org/10.1016/j.smrv.2017.06.011.

42. Li Y, Zhang X, Winkelman JW, Redline S, Hu FB, Stampfer M, et al. Association between insomnia symptoms and mortality: a prospective study of U.S. men. Circulation. 2014;129:737-46. https://doi.org/10.1161/CIRCULA TIONAHA.113.004500.

\section{Publisher's Note}

Springer Nature remains neutral with regard to jurisdictional claims in published maps and institutional affiliations.

Ready to submit your research? Choose BMC and benefit from:

- fast, convenient online submission

- thorough peer review by experienced researchers in your field

- rapid publication on acceptance

- support for research data, including large and complex data types

- gold Open Access which fosters wider collaboration and increased citations

- maximum visibility for your research: over $100 \mathrm{M}$ website views per year

At $\mathrm{BMC}$, research is always in progress.

Learn more biomedcentral.com/submissions 